\title{
Obstructive Sleep Apnea in Adults
}

\author{
Pralhad Prabhudesai ${ }^{1}$, Abha Pandey ${ }^{2}$
}

International Journal of Head and Neck Surgery (2019): 10.5005/jp-journals-10001-1365

\section{Case Description}

A 56 years male, smoker with BMI $42 \mathrm{~kg} / \mathrm{m}^{2}$ complaints of excessive snoring, choking spells, frequent night time awakenings for urination, evident daytime sleepiness with a recent history of near miss road traffic accident while driving on highway. He gave history of frequent alcohol consumption. He also had poorly controlled diabetes and hypertension. Despite all efforts, he was unable to lose weight. On examination, he had retrognathia, crowded oropharynx and neck circumference of 19 inches. PSG showed lowest $\mathrm{spO}_{2} 69 \%$, AHI 48/hr, REM AHI 62/hr.

\section{INTRODUCTION}

Obstructive sleep apnea (OSA) is a disorder where narrowing of upper airway leads to disturbance in normal ventilation during sleep. Decreased airflow due to repetitive complete or partial obstruction of the upper airway occurs with consequent progressive respiratory effort to overcome the obstruction. These obstructive respiratory events are typically associated with cortical microarousals leading to sleep fragmentation and consequent unrefreshing mornings ${ }^{1}$. Sleep is a restorative phenomenon for body. Instead in OSA patients, sleep becomes a stressful ordeal, the effect of which are borne by the patient not only in sleeping hours but also in the subsequent daytime hours in various ways. OSA has had its struggle with lack of awareness, significant expense of diagnosis and treatment and concerning bodily consequences. Awareness for this disease is still emerging, both amongst patient population and medical care givers across the world. In current scenario, the world has gathered enough literature to support a better understanding regarding the disease physiology, consequences, accompaniments and effect on overall morbidity and mortality. Table 1 shows the relevant definitions pertaining to OSA.

\section{Pathogenesis}

Pathogenesis of disease encompasses repeated narrowing of airway in sleep leading to oxygen desaturation, wide intrathoracic pressure swings, increased respiratory efforts and arousals. Recurrence of such events in body lead to multiple effects allover the body as depicted in Figure 1.

\section{Demographics}

Despite the rising awareness regarding OSA especially in the epidemic of obesity, the diseases by and large, continues to be widely underdiagnosed, across the world. The statistics regarding prevalence of OSA shows disparity due to variable basis of diagnosis. Symptoms, daytime sleepiness scores, different equipment or different cut off levels of $\mathrm{AHI}$ index have been chosen. Prevalence of OSA showed a rising trend overall but varies widely depending upon diagnostic criteria used. When only $\mathrm{AHI}$ was taken as basis of diagnosing OSA, prevalence in male was $22 \%(9-37 \%)$ and that in
1,2Department of Respiratory Diseases, Lilavati Hospital and Research Centre, Mumbai, Maharashtra, India

Corresponding Author: Pralhad Prabhudesai, Department of Respiratory Diseases, Lilavati Hospital and Research Centre, Mumbai, Maharashtra, India, e-mail: aumclinic@gmail.com

How to cite this article: Prabhudesai P, Pandey A. Obstructive Sleep Apnea in Adults. Int J Head Neck Surg 2019;10(1):22-30.

Source of support: Nil

Conflict of interest: None

Table 1: Definitions pertaining to OSA

\begin{tabular}{ll}
\hline Parameter & Definition \\
\hline Apnea & $\begin{array}{l}\text { Complete cessation of breathing for } \\
\text { more than } 10 \text { seconds }\end{array}$
\end{tabular}

Obstructive apnea Apnea with paradoxical abdominothoracic excursions

Central apnea Apnea with blunted abdomino- thoracic excursions

Hypopnea Reduction in airflow not amounting to complete cessation

Respiratory effort related Events with increased respiratory efforts arousals (RERA)

for more than 10 seconds along with arousals but do not meet the criteria for hypopnea

Apnea-hypopnea index Total number of Apnea and Hypopnea (AHI) per hour while in sleep

Respiratory distress index Total number of Apnea, hypopnea and (RDI) RERAs per hour while in sleep

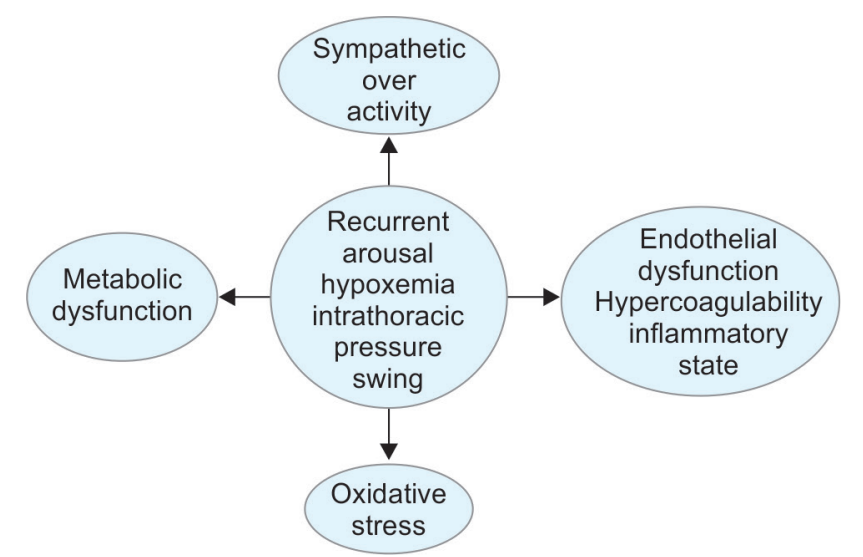

Fig. 1: Pathogenesis of OSA

females was $17 \%$ (4-50\%). ${ }^{2-7}$ However, when $\mathrm{AHI}>5 / \mathrm{hr}$ along with excessive daytime sleepiness was considered, prevalence in male dropped to $6 \%(3-18 \%)$ and in woman to $4 \%(1-17 \%)$. Subsequently,

(O) The Author(s). 2019 Open Access This article is distributed under the terms of the Creative Commons Attribution 4.0 International License (https://creativecommons. org/licenses/by-nc/4.0/), which permits unrestricted use, distribution, and non-commercial reproduction in any medium, provided you give appropriate credit to the original author(s) and the source, provide a link to the Creative Commons license, and indicate if changes were made. The Creative Commons Public Domain Dedication waiver (http://creativecommons.org/publicdomain/zero/1.0/) applies to the data made available in this article, unless otherwise stated. 
Obstructive Sleep Apnea in Adults

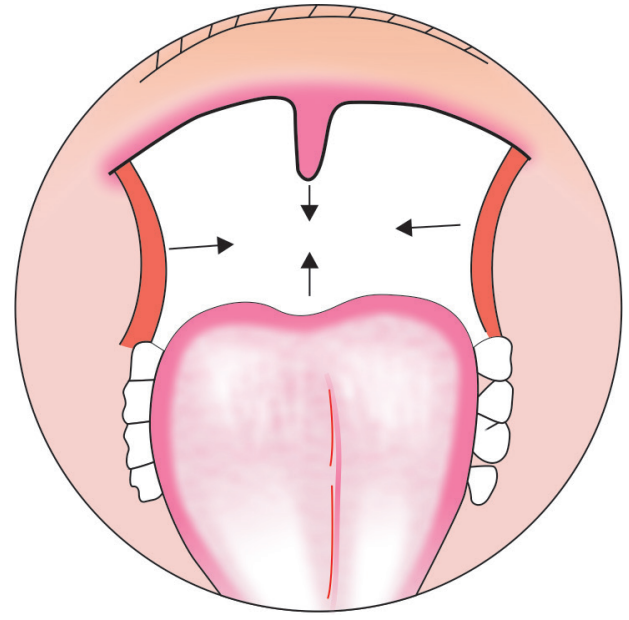

Fig. 2: Oropharyngeal space in OSA

prevalence of OSA showed a distinct rise in females as high as $50 \%{ }^{2}$ and up to $37 \%$ in males in postmenopausal age group. ${ }^{6}$

\section{RisK FACTORS}

There are certain factors which are known to predispose one to have OSA like advanced age, obesity, male gender, postmenopausal phase in females, acromegaly and craniofacial abnormality All these factors contribute to crowded oropharyngeal space while sleep as depicted in Figure 2.

Age

Prevalence increases with advancing age up to 60 years and thereafter plateau effect is seen. ${ }^{8}$

\section{Obesity}

Obesity has majorly contributed in OSA. The reason being the extraload of pharyngeal structures occluding the upper airway with a greater magnitude while lying supine. Loss of weight has favourably affected the severity of OSA. Despite all the emphasis on obesity associated with OSA, about one third of patients with OSA may not be obese. ${ }^{8}$

\section{Gender}

Prevalence of OSA in males is twice than that in females, in adults. This has been supported with the hormonal changes in males making their upper airway more collapsible, pattern of fat distribution and anatomy of upper airway. Females in postmenopausal age group show higher prevalence of OSA than in premenopausal phase due to hormonal influences in body. ${ }^{9}$ Importantly, presentation in females has been quite atypical than that in males and thus needs a higher index of suspicion. Prevalence of OSA becomes equal in males and females in postmenopausal age group.

\section{Acromegaly}

Excess of growth hormones by virtue of macroglossia and increased muscle mass in upper airway is known to predispose these patients to develop OSA. Approximately upto $75 \%$ of patients with acromegaly can have OSA.
Table 2: Symptoms of OSA

\begin{tabular}{ll}
\hline Daytime symptoms & Night time symptoms \\
\hline Unrefreshing sleep & Snoring-witnessed by bed partner \\
Morning headaches & Choking spells-witnessed by bed \\
Excessive daytime sleepiness & partner \\
Decreased cognition/ & Frequent awakening with dry \\
performance & mouth \\
Personality changes & Frequent urination \\
Erectile dysfunction & \\
Memory loss & \\
Decline in intellectual abilities &
\end{tabular}

\section{Comorbidities and Associations}

OSA has had its accompaniments like coronary artery disease, congestive heart failure, arrhythmias, refractory hypertension, type 2 diabetes, hypothyroidism, polycystic ovarian disease, chronic renal failure and neurocognitive disorders, anxiety and depression (being discussed in upcoming Issue 3 under Sleep Overlap Syndrome and Association with Other Medical Disorder).

\section{Clinical Presentation}

Classically, sleepiness in daytime and snoring with witnessed choking spells in night time in an obese patient are strongly suggestive of OSA. Usually, in early OSA patients and females, presentation may not be classical. Due to lack of awareness regarding the spectrum and consequences of disease, the symptoms are widely overlooked especially in the beginning. In advance stages, patient develops profound choking spells, hindrance in daily activities or more often poorly controlled comorbidities in the form of hypertension, diabetes or cardiac arrhythmias. It is very important to elicit history from patient as well as from their bed partner. Familial tendency for the disease should also be looked for. Presenting symptoms of OSA can be divided into daytime symptoms and night time symptoms (Table 2). Daytime sleepiness if noted should be scored with tools like Epworth sleepiness score. EDS should be carefully evaluated with the background of sleep history. In doubtful patients sleep diary and actigraphy can be helpful. EDS of OSA can be distinguished from that seen in narcolepsy by assessing the association with physical activity. In narcolepsy EDS can happen even with activity and while in OSA it is usually while sitting idle. Amongst nighttime symptom, snoring is very important. Primary snorers snore in a monotonous way in the same manner which may disappear on change of body positions while in OSA, snoring is associated with grunting sound which may become louder gradually and does not change with different body positions. The severity can lead to job related issues or road traffic accidents due to poor concentration. ${ }^{7}$ On physical examination, patients are commonly obese with increased neck circumference, short neck, craniofacial abnormality, retrognathia, micrognathia. Oral cavity shows crowded oropharynx with evident soft tissue deposition in neck, macroglossia with acromegaly or long floppy uvula. Signs of right heart failure may be present. Cluster of examination finding usually noted in OSA patients are obesity, increased neck girth, short neck, retrognathia and crowded oropharynx. Mallampati score is a helpful bedside tool for evaluating airway patency, which correlates very well with the 


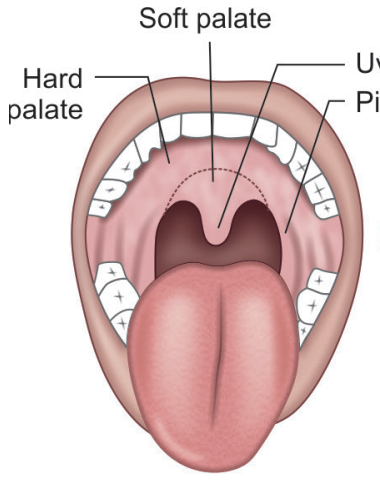

Class I

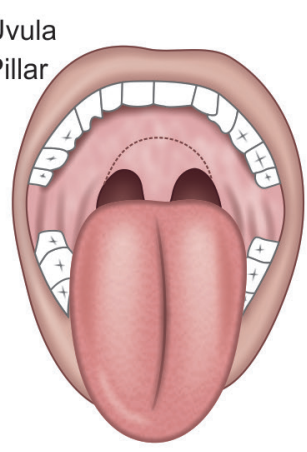

Class II

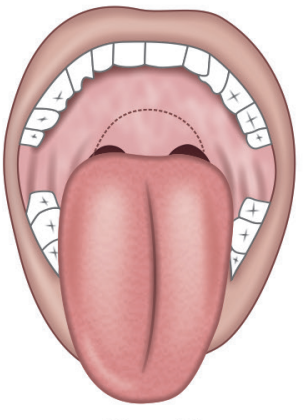

Class III

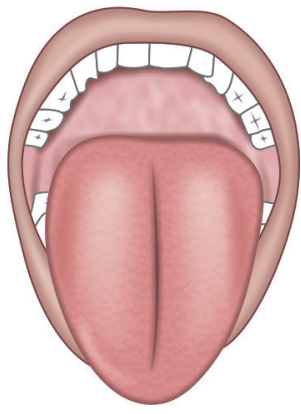

Class IV

Fig. 3: Mallampati score for patency of upper airway

\begin{tabular}{|c|c|c|}
\hline \multicolumn{3}{|c|}{ Suggestive clinical history } \\
\hline $\begin{array}{l}\text { Symptoms (daytime } \\
\text { and nighttime) }\end{array}$ & $\begin{array}{l}\text { Risk factor } \\
\text { evaluation }\end{array}$ & $\begin{array}{l}\text { Signs (obesity/neck girth/ } \\
\text { Mallampatti score) }\end{array}$ \\
\hline \multicolumn{3}{|c|}{$\nabla$} \\
\hline \multicolumn{3}{|c|}{ Useful predictive scores } \\
\hline $\begin{array}{l}\text { Epworth sleepiness } \\
\text { score (ESS) }\end{array}$ & $\begin{array}{l}\text { Stop bang } \\
\text { score }\end{array}$ & $\begin{array}{c}\text { Berlin's } \\
\text { questionnaire }\end{array}$ \\
\hline \multicolumn{3}{|c|}{$\checkmark$} \\
\hline \multicolumn{3}{|c|}{ Confirmatory polysomographic features } \\
\hline $\mathrm{AHI}>5 /$ hour & & $\begin{array}{l}\text { Significant desaturation } \\
\text { in sleep }\end{array}$ \\
\hline
\end{tabular}

Fig. 4: Diagnosis of OSA

possibility of upper airway narrowing in sleep (Fig. 3). Undiagnosed pulmonary hypertension or polycythemia warrants a PSG to rule out OSA. The struggle goes on between patient's perception of his daily living and physician's suspicion based on risk factors and symptomology, delaying the diagnosis of OSA.

\section{DiAgNOSIS}

Diagnosis of OSA comprises of a suggestive history, positive examination findings and confirmatory overnight polysomnography (PSG) (Fig. 4). In history, one should look for duration of symptoms, possible identifiable triggers like drugs and underlying medical disorders which should be addressed at priority. Also, an attempt to rule out mimics should be done. For example, sleep deprivation may be caused due to anxiety, depression disorder, diabetes, pain, prostatic symptoms, neuro stimulant drugs leading to excessive daytime sleepiness. Similarly, snoring can be due to pregnancy, nasal obstruction or congestion than OSA. Usually, supervised laboratorybased sleep study is the standard of practice for confident diagnosis of OSA in all scenarios. However, in resource limited settings or where there is strong likelihood of OSA and laboratory study is not feasible, home sleep analysis can be performed. There has been a strong recommendation that clinical tools, questionnaires and prediction algorithms should not be used to diagnose OSA in adults, in absence of polysomnographic or home sleep apnoea testing (HSAT). Detailed polysomnographic features have been discussed in Sleep Study Interpretation in OSA. Other supportive tests which can be helpful are sleep endoscopy, actigraphy and sleep diary. Sleep endoscopy has a role in diagnosis where dynamic
Table 3: Grading of OSA as per polysomnography results

\begin{tabular}{ll}
\hline Grade & AHI (events per hour) \\
\hline Mild & $5-15$ \\
Moderate & $15-30$ \\
Severe & $>30$ \\
\hline
\end{tabular}

collapse of upper airway is visualized under endoscopic guidance. Actigraphy is a noninvasive modality to gauge body movements, which should be cautiously used in OSA diagnosis. It is useful in insomnia, sleep phase disorders and circadian rhythm disorders. Sleep diary maintenance is another helpful adjunct in diagnosis, which should be used as supportive test only.

In our case, patient had classical history of obstructive sleep apnea and PSG showed severe nocturnal desaturation overnight with significantly high AHI/ REM AHI along with poorly controlled co morbidities. This is a classical case of severe OSA requiring immediate addressal.

\section{Grading Severity}

Severity of OSA is usually assessed by apnea hypopnea index (AHI) or respiratory distress index (RDI) scored over the sleep study report as mild, moderate and severe (Table 3). Additionally, extent of desaturation and duration of apnea are also important parameters often neglected. We feel that despite a lesser AHI index, if minimal $\mathrm{SpO}_{2}$ is significantly low the severity of disease should be graded higher. In presence of strong symptoms, the serial epochs should be read to screen whether the duration of apnea is prolonged causing a more significant fall in oxygen levels. Also, REM phase should be looked for a higher gravity of disease.

\section{Phenotypes}

Newer advances in the field brings in an outline of phenotypes in OSA like other diseases which may assist in better patient care (Table 4). Certain physiologic behaviour may dictate or dominate the OSA disease in patients. There are four main phenotypes identified as of now.

- Increased airway collapsibility: The pressure at which the airway collapses is called the pharyngeal critical closing pressure(Pcrit). It is taken as a surrogate marker to assess functional anatomy of upper airway in sleep. In normal healthy people Pcrit is negative. In situations where it is distinctly positive, airway is said to be highly collapsible. Usually in patients with OSA, Pcrit is positive or just above atompheric pressure. CPAP therapy which is gold 
Obstructive Sleep Apnea in Adults

Table 4: Remedies of different phenotypes of OSA

\begin{tabular}{|c|c|c|}
\hline Phenotypic traits & Measurement methods & Remarks/Remedies \\
\hline - Upper airway collapsibility & Pharyngeal pressure catheter and CPAP & $\begin{array}{l}\text { - Poor CPAP compliance in Pcrit negative } \\
\text { - Good CPAP compliance in pharyngeal critical pressure (Pcrit) } \\
\text { positive } \\
\text { Alternative therapy should be considered in Pcrit negative }\end{array}$ \\
\hline $\begin{array}{l}\text { - Upper airway poor muscle } \\
\text { responsiveness }\end{array}$ & $\begin{array}{l}\text { Epiglottic or esophageal pressure catheter } \\
\text { combined with PSG }\end{array}$ & $\begin{array}{l}\text { Targeted therapy: } \\
\text { - Hypoglossal nerve stimulation } \\
\text { - Pharmacotherapy: Desipramine, zolpidem } \\
\text { - Oropharyngeal muscle exercises } \\
\text { - Didgeridoo }\end{array}$ \\
\hline - Lower arousal threshold & Airway pressure catheter and PSG reading & $\begin{array}{l}\text { - Poor CPAP compliance } \\
\text { - Hypnotics }\end{array}$ \\
\hline - High loop gain & Nasal pressure signals & $\begin{array}{l}\text { Adjuncts to CPAP } \\
\text { - Oxygen therapy } \\
\text { - Carbonic anhydrase inhibitor } \\
\text { - Drug like zonisamine }\end{array}$ \\
\hline
\end{tabular}
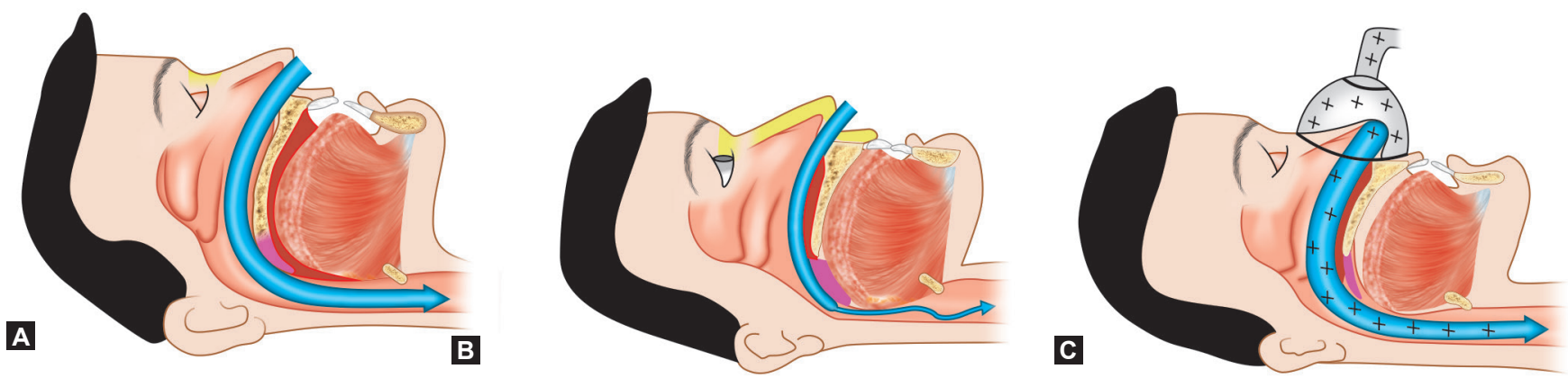

Figs 5 A to C: Airway patency diagram. (A) Normal airway; (B) Airway in OSA; (C) Airway in OSA with PAP

standard for treatment is found to be less effective in patients with negative Pcrit values.

- Poor responsiveness of upper airway muscle: Poor response of airway muscles to levels of carbon dioxide(ventilation) may be there. Neural control of upper airway muscle is a complex phenomenon. Genioglossus muscle is the largest muscle to stabilize the airway especially while inspiration. In $30 \%$ of patients, poor responsiveness of genioglossus muscle is the inciting cause for OSA leading to frequent awakening. This in turn leads to sleep instability, fragmentation, inability to enter deeper stages of sleep and unstable breathing.

- Lower arousal threshold: Arousal threshold dictates the interruption of sleep with awakening. In a subset of patients with lower arousal threshold, even relatively smaller intrathoracic pressure swings may lead to arousal. This subset of patient's land up with poor compliance to CPAP therapy.

- Poor stability offeedback controller system: Loop gain is defined as ventilator response to ventilator disturbances in body. One third of OSA patients are known to have high loop gain where they show increased ventilatory response to even a minimal change in $\mathrm{CO}_{2}$ levels thereby leading to frequent unwanted sleep disturbances. On the contrary, low loop gain is a phenomenon associated with Obesity hypoventilation syndrome where patients show suboptimal response to ventilator disturbance (high $\mathrm{CO}_{2}$ levels).

Phenotypes in OSA can be a mind storming advent in management of patients. However, it is yet struggling for validation and needs more research to be implemented in clinical practice as of now.

\section{MANAGEMENT}

Management of OSA depends upon multiple factors. OSA can have a component of upper airway anatomical compromise, neuromuscular instability or neuroventilator abnormality individually or in combination with varying extent. Importantly, they may complicate the cardiovascular or cerebrovascular functioning in the body. The decision to treat primarily depends upon presenting symptoms, severity of disease as per AHI by polysomnography, disturbance perceived by the bed partner and risk for cardiovascular or cerebrovascular consequences. There are several modalities available to treat the disease, from positive airway pressure (PAP), medical management, oral appliances to surgical interventions. PAP therapy is the standard of therapy for OSA. All the modalities can be clubbed together for management as add on therapy (therapy which can complement the CPAP therapy) or alternative therapy (for those who do not comply to CPAP or are unfit for (PAP). Treatment should be individualised than protocolized method.

\section{Positive Airway Pressure}

Positive airway pressure (PAP) therapy creates a pneumatic splint in upper airway to maintain the patency during sleep (Fig. 5). Thereby, preventing the soft tissue from collapsing which in turn reduces all the physiologic embarrassments in terms of apnoea, oxygen desaturation and frequent arousals. Immediate improvement in the level of alertness, concentration and memory has been noted with effective PAP therapy. However, neurocognitive symptoms may take 2 months also to resolve. Optimum pressures should 
Table 5: Milestones in course of administration of PAP therapy: inculcate doctor -patient partnership

\begin{tabular}{ll}
\hline At beginning & Stress on "Importance of therapy" \\
- How it helps \\
- Favorable consequences when used properly \\
- Dangerous consequences if not used properly \\
- Firm word for usage with empathy \\
- Assurance to handle problems in future \\
- Offer multiple options of interface \\
Stress on "Continuation of therapy" \\
- Monitor symptom improvement \\
- Check adherence to therapy \\
- Prompt addressal to issues related to therapy \\
- A word on equipment maintenance \\
- Ask for card readings in last 2 weeks \\
- Feedback on every visit
\end{tabular}

- Anonymous group should be formed to allow patients to interact regarding their experiences.

- Education should be provided regarding the disease and its management
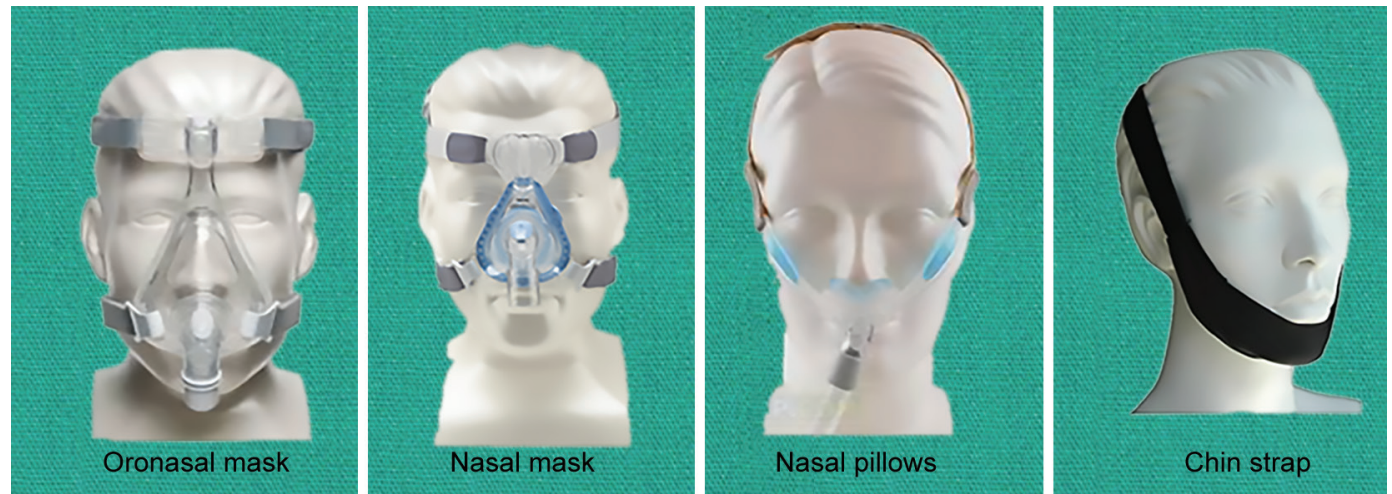

Fig. 6: Interfaces for PAP therapy

be decided by the PAP titration study report. Despite the best of benefits this therapy offers, compliance has been a major issue across the globe. Thus, it is prudent to give due importance to counseling and developing a partnership with patient. At the time of initiation of therapy, they should be explained what is OSA, how does it affect the body organs, why should it be treated, availability of therapeutic options to choose from and manageable side effects. Hazards of driving should be explained clearly in untreated patients, until they show improvement in symptoms later on. Care provider should be empathic towards patients and involve them at every step for successful treatment as mentioned in Table 5.

\section{Initiation of PAP Therapy}

PAP is recommended unanimously in all patients with an $\mathrm{AHI}$ of more than $15 /$ hour. However, there has been a conflict in prescribing PAP therapy in patients with mild OSA (AHI: $5-15 / h r)$. In patients with mild OSA, PAP therapy should be offered if patient has associated coronary artery disease, congestive cardiac failure, cerebrovascular failure or arrhythmia to herald the disease progression. Also, diseases with hypoventilation like neuromuscular disorders, parkinson disease or COPD need assessment for early initiation of PAP therapy. All patient once diagnosed with OSA, should undergo a PAP titration analysis. Unanimously, PAP initiation should be strictly done after understanding the optimum pressure levels as per the report than arbitrarily prescribing fixed pressures or Automatic mode. This holds a great importance in compliance of patients with PAP therapy eventually. We see that those who use PAP therapy regularly, are more compliant than those who use it intermittently.
Also, milder disease patients do not perceive positive effect of PAP therapy. In supine position, lung volumes comparatively are less and upper airway shape and size gets altered. In a subset of patients, where there is a documented positional variability of $\mathrm{AHI}$ (supine $\mathrm{AHI}>$ lateral decubitus AHI), certain measure can be incorporated to keep the patient in lateral position in sleeping hours. Interface selection should be strictly individualized (Fig. 6).

Common side effects of PAP therapy are dryness in throat, bloating sensation, eye irritation, nasal stuffiness, cold sensation in nose, skin rashes, inability to sleep, difficulty breathing out, sinus discomfort and claustrophobia. These concerns should be addressed at initial few visits. For persistent bothersome nasal congestion refractory to conservative therapy, nasal surgeries can be opted for, in a carefully selected patient. Dreaded complications in form of pneumothorax, pneumomediastinum and tympanic membrane rupture are extremely rare.

\section{Modes of PAP Therapy}

\section{Continuous PAP Therapy}

Continuous PAP (CPAP) therapy provides fixed pressure at the level of upper airway to keep it open while sleeping. PAP can be delivered either by a manual mode wherein constant pressure is delivered throughout the night or by an automatic mode where a dynamic assessment of airway lumen is done and appropriate pressures are delivered. The rationale of automatic mode is that pressures may vary not only over the same night but also over consecutive night hours and in different positions. Thus, automatic 
Obstructive Sleep Apnea in Adults

Table 6: Trouble shooting on CPAP therapy

\begin{tabular}{ll}
\hline Troubles & Solution \\
\hline Irritation in eyes & Mask apposition \\
Dryness of mouth & Humidifier \\
Cold sensation at nose & Humidifier \\
Snoring on CPAP & Chin strap \\
Excessive mouth leaks & Chin strap \\
Excessive noise & Chin strap, reduce pressures or use ear \\
& plugs \\
Nasal congestion & Local corticosteroids \\
Nasal dryness & Local saline drops / Humidifier \\
Claustrophobia & Change the interface \\
& Oro nasal $\rightarrow$ nasal mask $\rightarrow$ nasal pil- \\
Increased resistance felt & $\begin{array}{l}\text { lows } \\
\text { on expiration }\end{array}$ \\
\hline
\end{tabular}

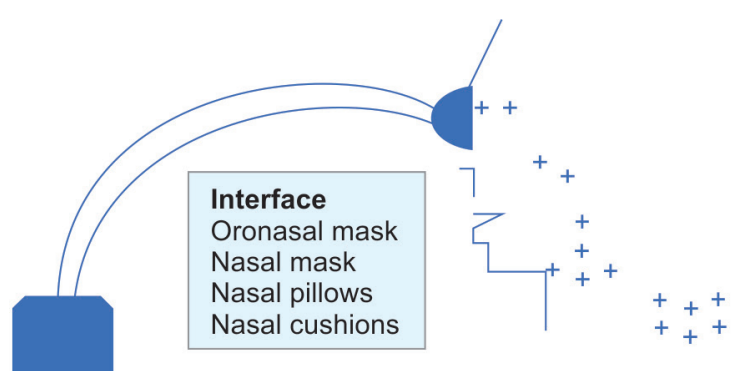

\begin{tabular}{|l|}
\hline Machine \\
Mode \\
Humidifier \\
Setting adjustment \\
Portability \\
Cost
\end{tabular}

\begin{tabular}{|l|}
\hline Patient \\
Education \\
Compliance \\
Build partnership \\
Anonymous \\
Symptom monitoring
\end{tabular}

Fig. 7: Successful PAP therapy should address machine, interface and patient related factors

CPAP (Auto CPAP) allows the pressure adjustment according to the requirements like after alcohol consumption, sedatives, etc which may cause transient rise in requirement. There has been no statistical difference in treatment success or compliance between the two modes in general. However, for patients with high pressure requirement Auto CPAP should be preferred. On the contrary, Auto CPAP should not be used in morbidly obese patients, overlap with COPD and in patients with concomitant heart failure. On regular follow ups, patient should be asked for remnant snoring or worsening in symptoms despite the therapy. Success with PAP therapy needs timely troubeshooting (Table 6) and due attention to various aspects (Fig. 7).

\section{Bi-level PAP}

Bi-level PAP (BPAP) mode delivers separate pressures in inhalation (IPAP) and exhalation phase (EPAP) of breathing, wherein exhalation pressure is lesser than the inhalation pressure. Difference between the IPAP and EPAP is called pressure support, which decides the tidal volume that patient generates. This mode is not the standard of therapy at the outset of PAP therapy. However, in patients with associated hypoventilation due to overlap with COPD, BPAP support may be beneficial. Also, in a subset with obesity hypoventilation syndrome or complex apnea or those patients who are willing to take PAP therapy but are unable to tolerate CPAP mode, BPAP may be helpful. There has been no statistical advantage for opting BPAP mode over CPAP mode as yet. Importantly, BPAP titration should be done carefully by a skilled person before starting the therapy for optimum pressures.

\section{Assisted Servo Ventilation}

Assisted servo ventilation (ASV) mode delivers varying inspiratory pressures and a constant lower expiratory pressure to the patient. This mode has been helpful in patients with mixed or complex apnea where patient has a component of central apnea along with OSA either accompanying at the time of diagnosis (mixed apnea) or emerging after initiation of PAP therapy (complex sleep apnea) for OSA. No statistical benefits over CPAP therapy have emerged as yet.

\section{Volume-assured Pressure Support}

Volume-assured pressure support (VAPS) is a variable bilevel PAP therapy mode that allows the target volume or ventilation to be pre-programed, thereby allowing more definitive control over ventilation. Respironics AVAPS (average VAPS) and ResMed iVAPS (intelligent VAPS) adjust the PS in order to maintain target average ventilation over several breaths. This mode of PAP is often helpful for patients with respiratory insufficiency due to neuromuscular and restrictive conditions in which respiratory effort varies during sleep or who need PAP during the day, COPD patients with risk of hypoventilation, and for obesity hypoventilation patients who may need compensation based on positional and sleep stage changes.

With any mode given to patients at times some fine tuning is required in the settings to make the therapy more comfortable. If patient feel excessive pressures, the set pressure can be reduced or ramp settings can be tried where the patient would receive higher pressures gradually. Chin strap can be added to patients with persistent bothersome air leaks. Solutions to concerns should be provided as and when required (Table 6).

\section{Compliance Issues}

Adherence to PAP therapy is the biggest challenge faced by sleep physicians today. Compliance to PAP therapy is a consistent pitfall over ages. Less than 4 hours of use overnight is considered as nonadherent to therapy. Unfortunately, $29-83 \%$ of patients are found to be non- adherent which is alarming. ${ }^{12}$ All attempts should be taken to enhance the same. Like any other ailment, OSA patients also need regular OPD follow up to address to their concerns and ensure PAP usage. If need arises, where all efforts to improve the compliance have failed, alternate therapies should be offered. Also, understanding the several phenotypes better there can be a domain where PAP therapy may not be genuinely helpful. There has been a concept of PAP NAP therapy where patients who have OSA along with insomnia issues and are really skeptical for PAP therapy, trial of PAP therapy is given in their daytime nap.

\section{Ancillary Measures}

In addition to PAP therapy, more can be done to improve overall outcome. Some ancillary measures should be considered irrespective of severity of OSA in all the patients.

Weight reduction has been an established measure for reducing AHI when used appropriately in obese subset of patients. Strict dietary restrictions and exercise program should be followed for gradual effective weight loss. Reduction of $10 \%$ of body weight, has shown to reduce $\mathrm{AHI}$ by approximately $26 \%$. Bariatric surgery has 


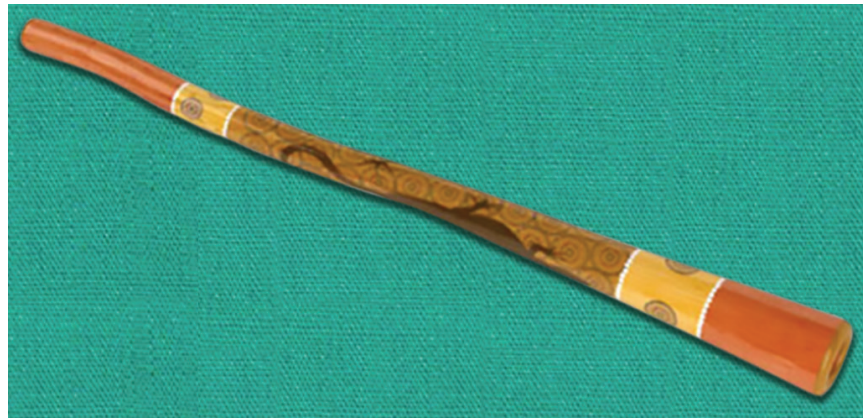

Fig. 8: Didgeridoo—A musical instrument

a role in weight reduction and has had a definitive role in bringing down the AHI in morbidly obese patients. However, they still have to be motivated enough to maintain the weight thereafter.

Body position affecting dynamics in upper airway patency has been well studied in OSA patients. It has been proven that supine position predisposes for a higher $\mathrm{AHI}$ than lateral recumbent position. Thus, for those patients who have documented evidence of higher AHI in supine position, positional therapy should be opted for. Tennis ball vest or sewing a tennis ball over the back side of the shirt and verbal alarms usually help not to acquire supine position while sleeping. One can be asked to sleep in elevated head position by raising the head end. However, this is only an adjunct to an effective PAP therapy.

Pharyngeal muscle exercises help tone pharyngeal muscles which in turn helps to keep the airway open while sleeping. Several exercising illustrations have appeared basically to stretch open the narrowed airway lumen. Didgeridoo is an ancient musical instrument, which has regained its charm with emerging epidemic of OSA. Studies have shown that there has been benefits of using this therapy in mild to moderate grade OSA patients. Although there has been lacuna in literature, but available data show that regular didgeridoo playing may reduce snoring and excessive daytime somnolence. This instrument works on principles of circular breathing. This requires breathing in through the nose while simultaneously blowing out the air from mouth using the tongue and cheeks. Lips vibrate continuously to produce a drone. Circular breathing is controlled by upper airway and leads to pharyngeal muscle exercise. This stiffens the pharynx and prevents airway collapse. However, it cannot be done by an obese patient, those with structural airway defect or central sleep apnea (Fig. 8).

Hypoglossal nerve stimulation (HGNS) helps by increasing the output of hypoglossal nerve which supplies primarily genioglossus muscle and thereby help in opening up the hypopharyngeal as well as retropalatal airway better. It is comprised of three parts namely, stimulation cuff electrode, pleural pressure sensing lead and an implantable pulse generator (IPG). Neuromodulation of airway has emerged as a promising therapy in CPAP intolerant patients. Neural stimulation leads to restoration of upper airway muscle tone while sleeping. It is a one time procedure but is titratable like CPAP. HGNS cannot be used in obese patients like with $B M I>32 \mathrm{~kg} / \mathrm{m}^{2}$, due to inconsistent results. ${ }^{10}$

\section{Other Therapies}

\section{Expiratory Nasal Pressure Therapy}

Expiratory nasal pressure is created with the help of a mechanical valve device which is snuggly fitted at both the nasal openings

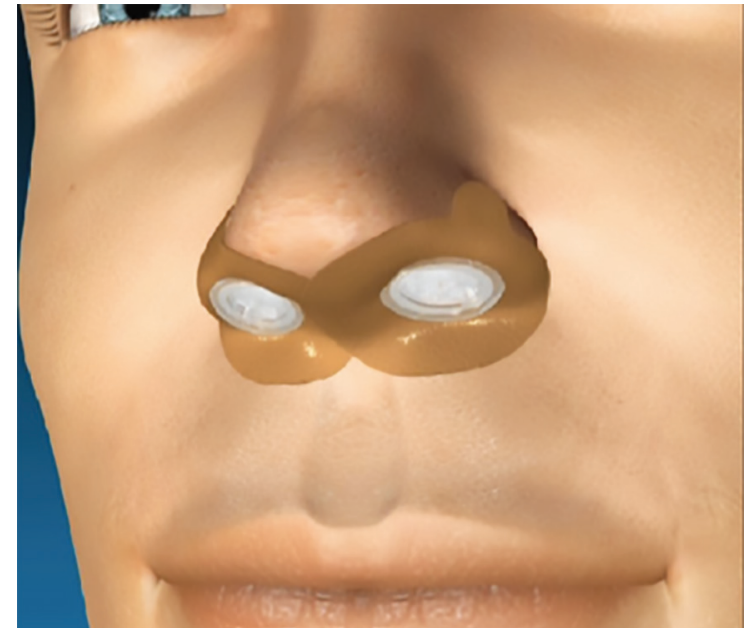

Figure 9: Expiratory nasal pressure valve

with the help of an adhesive. It allows for inhalation with minimal resistance but offers significant resistance to exhalation thereby creating a positive pressure in the upper airway while sleeping. Compliance with the therapy has been more than $80 \%$, which is significant. This therapy still needs more data support but can turn out to be a promising alternative option to PAP therapy in future especially for frequent travellers (Fig. 9).

\section{Oral Pressure Therapy}

A negative pressure is created in the oral cavity with the help of a mouthpiece which is placed over the teeth connected to a console. This subtle suction pressure (approximately $20 \mathrm{~cm}$ of water) is enough to pull the soft palate anteriorly, stabilizing the tongue and opening up the airway lumen behind. Different sizes of mouth piece are available. Literature supports some strong responders but still a significant number of non-responders to this noninvasive pressure system are known. (Fig. 10)

\section{High-flow Nasal Therapy}

It is a lower positive airway pressure therapy wherein a nasal cannula is used to deliver pressure in the upper airway (Fig. 11). The best of the benefits are obtained when mouth is closed and leak around the cannula is minimum. However, it needs more elaborate study to analyze the quantum of benefits, variability with mouth opened state and effectiveness in OSA consequences at a large level. ${ }^{11}$

\section{Venous Compression in Legs}

Rostral shift of fluid from the lower limb has shown to increase the engorgement in the neck in night leading to worsened pharyngeal obstruction while sleep. This is a fact associated with both OSA and CSA patients. In OSA patients especially men having sedentary lifestyle, the accumulated fluid in legs can be considerable. While lying recumbent in sleep, redistribution of this fluid would worsen the upper airway mechanics. Thus, daytime usage of leg stockings to avoid this fluid collection may curtail down the additional load on already compromised upper airway.

Among general measures one should always try to abide to a sound sleep hygiene practices as described in Sleep and HealthAn Introduction. Alcohol and tobacco consumption especially close to sleeping time should be best avoided. Hypnotics and sedatives which are known to aggravate OSA in patients should 
Obstructive Sleep Apnea in Adults

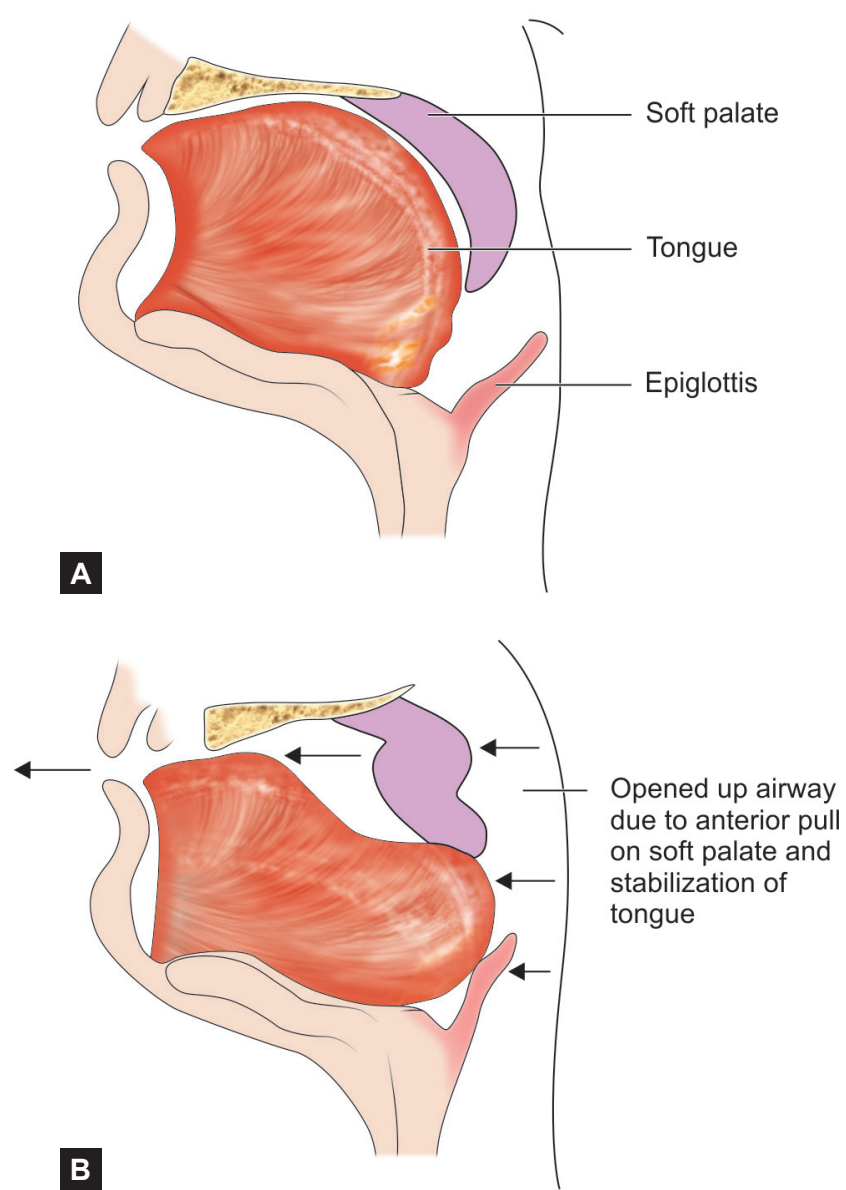

Figs $10 A$ and B: Dilatation of upper airway with use of OPT (A) Upper airway anatomy in OSA; (B) Upper airway anatomy with OPT be avoided. Oxygen therapy is not the standard of therapy as the problem in OSA is collapsibility and not the chemoreceptor alteration or inherent oxygen deficit. On the contrary giving oxygen can be deleterious as it may prolong the apnea by delaying the arousal. This can have poor cardiovascular side effects. However, in a peripheral setup where CPAP availability or affordability is an issue oxygen therapy can be tried. Oral appliances and surgery has been explained in dental appliances and mandibular advancement devices in OSA.

Antisnoring devices are often used as are easily available. These devices are strongly contraindicated in OSA patients and should be used only after documenting no OSA on PSG by a sleep physician only.

\section{Medical Management ${ }^{12}$}

None of the pharmacological agents have emerged as a replacement of standard PAP therapy as of now. Patients with underlying allergic rhinitis should be treated with nasal corticosteroids on a regular basis. Some neurostimulants like theophyllines or acetazolamides and desipramine have been studied but need more literature support to incorporate them in treatment protocol. However, in those patients who continue to suffer with excessive daytime sleepiness despite effective CPAP therapy, modafinil can be used when all other possible causes have been ruled out under supervision of sleep physician.

\section{Pearls of Wisdom}

- OSA is a sleep disordered breathing which has significant systemic consequences.

- Diagnosis needs suggestive clinical presentation and polysomnography.

- CPAP is the standard of therapy for OSA.

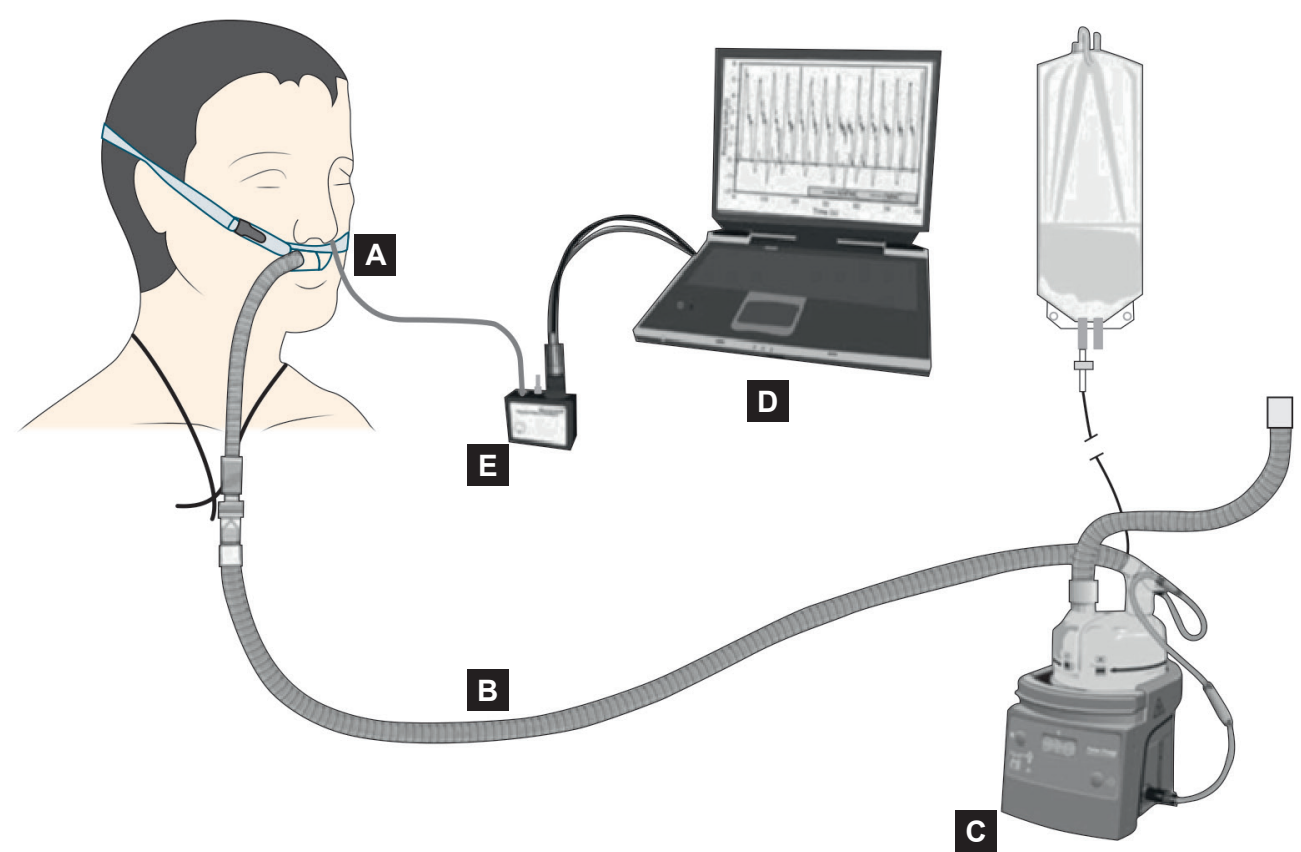

Figs 11 A to E: High-flow nasal therapy setup for OSA . (A) Nasal cannula; (B) Heated delivery tube; (C) Humidifier; (D) Monitor; (E) Air and oxygen blender. 
- In mild OSA if significant desaturation and cerebrovascular comorbidities are found, CPAP should be initiated.

- Side effects of OSA are way more devastating than side effects of PAP therapy.

- Never suppress snoring in a patient without ruling out underlying OSA.

- Partnership with patient in the management of OSA can take care of many roadblocks.

- Repeated education, addressal of issues with PAP therapy should be done on follow-up visits.

- Give due attention to remnant sleepiness despite CPAP therapy to look for other sleep disorders.

- Give importance to sleep hygiene in all patients.

\section{References}

1. Caples SM, Gami AS, et al. Obstructive sleep apnea. Ann Intern Med 2005;142:187-197

2. Franklin KA, Sahlin C, et al. Sleep apnoea is a common occurrence in females. Eur Respir J 2013;41:610-615.

3. Ip MS, Lam B, et al. A community study of sleep-disordered breathing in middle-aged Chinese women in Hong Kong: prevalence and gender differences. Chest 2004;125:127-134.

4. Udwadia ZF, Doshi AV, et al. Prevalence of sleep-disordered breathing and sleep apnea in middle- aged urban Indian men. Am J Respir Crit Care Med 2004;169:168-173.
5. Sharma SK, Kumpawat $\mathrm{S}$, et al. Prevalence and risk factors of obstructive sleep apnea syndrome in a population of Delhi, India. Chest 2006;130:149-156.

6. Nakayama-Ashida $Y$, Takegami M, et al. Sleep- disordered breathing in the usual lifestyle setting as detected with home monitoring in a population of working men in Japan. Sleep 2008;31:419-425.

7. Jackson ML, Howard ME, et al. Cognition and daytime functioning in sleep-related breathing disorders. Prog Brain Res. 2011;190:53-68.

8. Svensson $M$, Lindberg $E$, et al. Risk factors associated with snoring in women with special emphasis on body mass index: a populationbased study. Chest 2006;129:933-941.

9. Young T, Finn L, et al. Sleep disordered breathing and mortality: eighteen-year follow-up of the Wisconsin sleep cohort. Sleep 2008;31:1071-1078.

10. Sung ok Hong, Yu -Feng Chen, et al. Hypoglossal nerve stimulation for treatment of obstructive sleep apnea (OSA): a primer for oral and maxillofacial surgeons. Maxillofacial Plastic and Reconstructive Surgery 2017;39:27.

11. Parke R1, McGuinness S, et al. Nasal high-flow therapy delivers low level positive airway pressure. British Journal of Anaesthesia 2009;103 (6): 886-890.

12. Veasey SC, Guilleminault C, et al. Medical therapy for obstructive sleep apnea: a review by the medical therapy for obstructive sleep apnea task force of the standards of practice committee of the American Academy of Sleep Medicine. Sleep 2006;29(8): 1036-1044. 